Cahiers de Narratologie

Sérialité narrative. Enjeux esthétiques et économiques

\title{
Le roman-feuilleton ou l'écriture mercenaire : l'exemple des Mystères de Paris
}

Anaïs Goudmand

\section{(2) OpenEdition}

\section{Journals}

Electronic version

URL: http://journals.openedition.org/narratologie/7589

DOI: 10.4000/narratologie.7589

ISSN: 1765-307X

Publisher

LIRCES

Electronic reference

Anaiis Goudmand, «Le roman-feuilleton ou l'écriture mercenaire : l'exemple des Mystères de Paris », Cahiers de Narratologie [Online], 31 | 2016, Online since 22 December 2016, connection on 19 April 2019. URL : http://journals.openedition.org/narratologie/7589; DOI : 10.4000/narratologie.7589

This text was automatically generated on 19 April 2019

Article L.111-1 du Code de la propriété intellectuelle. 


\title{
Le roman-feuilleton ou l'écriture mercenaire : l'exemple des Mystères de Paris
}

\author{
Anaïs Goudmand
}

1 L'histoire de la presse française est marquée dans les années 1830 par un bouleversement majeur: en 1836, Émile de Girardin lance La Presse, journal qui repose sur une double innovation. En premier lieu, l'introduction de l'«annonce» (équivalent de l'actuelle publicité) permet de réduire de moitié le coût de l'abonnement par rapport aux publications concurrentes ( 40 Francs au lieu de 80 Francs $^{1}$ ). Ensuite, la publication de romans en tranches quotidiennes dans les colonnes du journal attire un nouveau public et garantit ainsi aux annonceurs un retour sur investissement satisfaisant. Le procédé du «feuilleton-roman», rebaptisé "roman-feuilleton» par la suite, se généralise dans la décennie suivante et devient quasiment incontournable pour tous les romanciers : entre 1836 et 1845, la plupart des journaux qui appliquent la formule initiée par La Presse voient leur tirage doubler, notamment grâce au succès du roman-feuilleton, qui contribue pour une large part à l'élargissement du lectorat. Le statut des auteurs se trouve radicalement modifié: le système de mécénat, dans lequel l'écrivain était pensionné, cède progressivement sous le développement de l'écriture salariée. Le roman-feuilleton est donc emblématique de l'entrée de la littérature dans le capitalisme puisqu'il met en œuvre une articulation inédite des fonctions esthétique et économique de la littérature, qui fait ainsi son entrée dans l'« ère médiatique ", suivant l'expression de Marie-Ève Therenty et Alain Vaillant (2001). Le récit ne peut dès lors être pensé en dehors du contexte de production qui l'informe, dans la mesure où le roman-feuilleton est immédiatement conçu par les auteurs en fonction de la publication journalière et où la continuation de l'œuvre est immédiatement dépendante de son succès auprès du public. L'objectif de rentabilité immédiat pousse les auteurs à multiplier les "effets " pour harponner efficacement le lecteur et le pousser ainsi à poursuivre la lecture lors de la livraison suivante, ce qui explique la dévalorisation durable des procédés narratifs hérités du feuilleton. Ainsi, le roman-feuilleton suscite très tôt de vives réactions de la part des 
élites intellectuelles, qui s'inquiètent des effets néfastes d'une démocratisation de la littérature et multiplient les stratégies de dévalorisation symbolique, à l'image du pamphlet de Sainte-Beuve contre la "littérature industrielle ", publié dès 1939, qui prône une séparation sans équivoque des domaines artistique et économique, et donc une sacralisation de l'espace littéraire : «Quelle que soit la légitimité stricte du fond, n'est-il pas triste pour les lettres en général que leur condition matérielle et leur préoccupation besogneuse en viennent à ce degré d'organisation et de publicité ? " (Sainte-Beuve, in Dumasy 1999: 38-39). En effet, ce mode de publication constitue déjà, avant même la scission du champ littéraire analysée par Bourdieu (1992), une mise à l'épreuve d'une conception désintéressée de la littérature. Gaschon de Molènes assimile le travail du feuilletoniste au travail à la chaîne :

On dit qu'il y a dans les ateliers d'arts mécaniques une façon de distribuer le travail qui le rend plus facile et plus rapide : s'il s'agit de faire un carrosse, l'un est chargé des roues, l'autre des ressorts, un troisième du vernis et des dorures. Nous serions vraiment tenté de croire, en voyant certaines œuvres qui se disent pourtant des œuvres d'intelligence, qu'il y a des fabriques littéraires où l'on a recours à ces procédés. (Gaschon de Molènes, in Dumasy 1999 : 157)

2 Suivant une rhétorique déjà bien rodée, et qui persiste de nos jours à l'encontre de la télévision ou le jeu vidéo, le roman-feuilleton serait une machine à produire du bénéfice en décérébrant des lecteurs incapables du moindre recul critique. Dans ce contexte, Eugène Sue est parvenu à produire un récit particulièrement addictif, ce qui n'a pas manqué de susciter les foudres de ses détracteurs, mais également de fasciner certains de ses contemporains, dont Théophile Gautier :

Des malades ont attendu pour mourir la fin des Mystères de Paris; le magique La suite à demain les entraînait de jour en jour, et la mort comprenait qu'ils ne seraient pas tranquilles tant qu'ils ne connaissaient pas le dénouement de cette bizarre épopée. (Gautier : 1844)

3 Cette étude vise à analyser les conséquences narratives de la publication en tranches quotidiennes en nous intéressant aux modalités de la tension narrative en régime feuilletonnesque au moment du premier âge d'or du roman-feuilleton. Nous nous attacherons plus particulièrement à l'étude d'exemples tirés des Mystères de Paris. Parus entre 1842 et 1843 dans Le Journal des Débats et considérés comme l'un des premiers bestsellers de la littérature française, Les Mystères de Paris d'Eugène Sue concentrent tous les défauts supposés que réprouvent les adversaires du feuilleton (incohérences, pauvreté du style,...). Ils racontent les exploits de Rodolphe de Gerolstein, grand-duc allemand qui se fait passer pour un ouvrier afin de se mêler aux populations des bas-fonds parisiens et de récompenser les pauvres vertueux. Il rencontre ainsi des dizaines de personnages, dont Fleur-de-Marie, jeune prostituée, et le Chourineur, ancien criminel devenu boucher. Nous espérons montrer qu'il serait un peu rapide de résumer les effets du roman-feuilleton à de simples procédés commerciaux et de considérer que la valeur esthétique et éthique du récit de fiction serait inversement proportionnelle à sa fonction économique. Nous le verrons, plus on avance dans le récit, plus l'impératif de la publication journalière est crucial pour comprendre sa structuration, ce qui ne signifie pas pour autant une mise à l'écart des autres fonctions de la fiction, bien au contraire: outre la mise en place progressive des techniques narratives du récit sériel, on trouve dans l'œuvre de Sue des éléments de réflexion sur la fonction éthique du récit de fiction. Nous analyserons un motif narratif récurrent dans les Mystères, qui constitue un archétype romanesque emblématique d'une tension paroxystique : la scène de sauvetage, dans laquelle le héros 
ou l'héroïne en danger de mort est sauvé in extremis par un autre personnage. Nous verrons que la mise en place des stratégies narratives du feuilleton est progressive et corrélée au rythme d'écriture.

\section{Éléments de poétique du feuilleton}

4 La poétique du feuilleton repose sur des stratégies récurrentes qui permettent de maintenir l'intérêt du lecteur. En premier lieu, il s'agit de multiplier les points d'accroche et ainsi de proposer un récit prolongeable à merci, fondé sur ce que Régis Messac nomme une " esthétique divergente », qu'il oppose à l' « esthétique convergente » des short stories publiées mensuellement et non quotidiennement dans les magazines anglais :

Dans un journal quotidien [...], il s'agit de trouver chaque jour un nouveau motif d'intérêt et il n'y aura aucun inconvénient, loin de là, à laisser en suspens une curiosité qui n'aura pas le temps de s'émousser en vingt-quatre heures. Au lieu d'une esthétique convergente, à la Poe, le feuilleton aura donc une esthétique divergente; au lieu de chercher l'unité d'intérêt, on y cherchera la multiplicité d'intérêt. [...] [o]n peut dire qu'un vrai feuilleton n'a jamais de fin : s'il est bon, il a seulement des suites. (Messac 2011 [1929] : 332)

5 Ensuite, le roman-feuilleton se caractérise par l'interruption temporaire du récit entre deux segments. En contraste avec le découpage chapitral, la séparation n'est pas seulement matérielle, mais aussi temporelle :

Les livraisons se trouvent non seulement isolées dans l'espace, mais séparées dans le temps; la fin de chaque livraison équivaut à celle de l'« œuvre » elle-même, telle qu'elle existe alors dans sa version publique - et le plus souvent dans sa version privée. Il est impossible d'en poursuivre la lecture tant que n'est pas parue la livraison suivante, c'est-à-dire tant que n'est pas expiré un délai, sinon imprécis (comme dans les romans périodiques des XVII ${ }^{\mathrm{e}}$ et XVIII ${ }^{\mathrm{e}}$ siècles), tantôt expressément formulé (comme dans le roman-feuilleton ou le serial dickensien), mais toujours bien réel, et absolument irréductible. (Dionne 2008: 86)

Conséquences en termes rhétoriques et fonctionnels: la «réticence» de l'intrigue (Barthes 1973) est redoublée, surexploitée par l'interruption temporaire du récit, dans le but évident d'accroître le désir du lecteur de connaître la suite. La mise en intrigue est dès lors « sur-réticente », en quelque sorte : la série se caractérise par une tension intermittente , reposant sur une narration discontinue, interrompue à la fin de chaque unité, par opposition à la tension continue qui caractérise les autres types de récit, et le lecteur ne maîtrise pas les pauses dans l'actualisation du récit. Dès lors, la coupe revêt une importance fondamentale, comme l'a noté René Guise : « Le premier aspect particulier de la technique du roman-feuilleton est l'art du découpage » (1964: 300). Ce procédé a été observé - et moqué - très tôt par les contemporains : on le retrouve par exemple formulé par un rédacteur en chef qui dicte ses consignes à un futur feuilletoniste dans le roman parodique de Louis Reybaud, Jérôme Paturot à la recherche d'une position sociale, publié en feuilletons dans Le Constitutionnel en 1842, au même moment que Les Mystères de Paris :

C'est surtout dans la coupe, môsieur, que le vrai feuilletoniste se retrouve. Il faut que chaque numéro tombe bien, qu'il tienne au suivant par une espèce de cordon ombilical, qu'il inspire, qu'il donne le désir, l'impatience de lire de suite. Vous parliez d'art, tout à l'heure; l'art, le voilà. C'est l'art de se faire désirer, de se faire attendre. Vous avez, je suppose, un M. Arthur auquel votre public s'intéresse. [...] À la fin de chaque feuilleton, une situation critique, un mot mystérieux, et Arthur, toujours Arthur au bout! Plus le public aura mordu à votre Arthur, plus vous devez en tirer parti, le lui présenter comme une amorce. (Reybaud 1844 [1842] : 78-79) 
7 Le rédacteur en chef donne ensuite à son disciple un exemple ce qu'il appelle un effet «très nouveau » (Reybaud 1844 [1842] : 80) avant de se lancer dans une explication de texte en bonne et due forme :

«[...] Éthelgide, épouvantée, se jeta sur son lit et chercha à se faire un rempart de ses rideaux; mais quel fut son effroi quand elle vit sortir des parois du mur qui faisait face à sa couche un bras nu et une main livide tenant par les cheveux une tête sanglante et défigurée.

Quelle était cette main !!! Quelle était cette tête !!!»

(La suite au prochain numéro.)

- Voilà, môsieur, ce que j'appelle arrêter un feuilleton. C'est-à-dire que sur deux millions de lecteurs, il n'en est pas un seul qui ne voudra savoir ce que c'est que cette tête si hardiment suspendue entre deux numéros. On peut qualifier le moyen de triomphant. (Reybaud 1844 [1842] : 81-82)

On aura reconnu dans cet exemple fictif ce qu'on appelle aujourd'hui un "cliffhanger ", effet familier qui repose sur l'interruption du récit au moment où la tension est à son comble (climax) et qui est devenu l'emblème de la commercialité racoleuse du feuilleton. Raphaël Baroni a montré que la déconsidération durable du suspense était liée à sa fonction d'argument de vente, notamment dans le cas des récits sériels :

Le cas du roman-feuilleton est un peu particulier, car la fonction publicitaire de la tension narrative est remplie par le découpage du récit, qui exerce une forme de chantage sur le consommateur, et le pousse à acheter le quotidien du lendemain pour connaître la suite de l'histoire - et, réciproquement, le journal devient un support publicitaire pour le roman qui paraîtra ultérieurement dans sa version intégrale (Baroni 2004).

Il souligne ensuite que dans le cas des récits unifiés, « on ne peut faire l'expérience de la tension narrative que dans la mesure où le produit (littéraire ou cinématographique) a déjà été acheté » (Baroni 2004) : l'acte de consommation précède l'actualisation du récit elle-même, c'est essentiellement le paratexte qui prend en charge la dimension publicitaire, en explicitant la promesse d'une tension narrative. À l'inverse, les récits sériels maintiennent durablement les récepteurs dans leur statut de consommateur, dans le sens où chaque épisode fait la réclame du suivant, ce qui est certainement l'une des fonctions primordiales du cliffhanger. Si l'on définit le cliffhanger à partir de l'effet qu'il provoque chez le lecteur, on peut considérer que sa caractéristique la plus notable consiste dans le fait qu'il structure une tension qui continue à se faire ressentir après l'actualisation de la livraison. Pour reprendre la terminologie de Baroni (2007), tout récit structure une incertitude qui se traduit par un effet de suspense (lorsque l'incertitude porte sur l'avenir de l'histoire) ou de curiosité (lorsque l'incertitude porte sur le passé ou le présent de l'histoire) qui s'articule à une activité cognitive de l'interprète, encouragé à produire des pronostics (dans le cas du suspense) ou des diagnostics (dans le cas de la curiosité). L'interruption temporaire du récit au moment du climax tend dès lors à intensifier le désir de l'interprète de connaître la suite : privé du dénouement attendu, et donc du soulagement qui en résulte, celui-ci continue à anticiper les scénarios possibles pendant le laps de temps qui sépare les épisodes. L'intensification de l'activité imaginative par une coupe stratégique a été notée par Wolfgang Iser, qui soulignait que, dans le roman-feuilleton, "(l)e lecteur est obligé de faire travailler son imagination de façon plus intensive que dans le cas de la lecture d'un livre » (1976: 333), à cause du délai 
imposé avant le dénouement attendu : «la forme du feuilleton multiplie les disjonctions ou donne un impact plus grand aux disjonctions existantes en raison de la coupure " (1976 : 334). Il y a donc désolidarisation de la tension représentée (ou, plus exactement, de la représentation d'une situation tendue) et de la tension ressentie par l'interprète, qui est augmentée par l'impossibilité d'avoir immédiatement accès au fin mot de l'histoire. En d'autres termes, la «dysphorie passionnante» (Baroni 2007: 131) qui caractérise la tension narrative est à son comble en l'absence du récit qui la structure : il y a cliffhanger lorsque l'activité anticipatrice du récepteur atteint son intensité maximale après la fin de l'épisode (voir illustration 1). Bien entendu, cette tension ne persiste pas à un niveau identique pour le récepteur pendant toute la durée qui sépare la diffusion des épisodes : on peut raisonnablement supposer qu'elle est plus forte dans les instants qui suivent immédiatement la fin du premier épisode et ceux qui précèdent la livraison suivante : la chute de la courbe de la tension ressentie dans le graphique montre le désengagement momentané du lecteur vis-à-vis du récit, lorsqu'il reprend ses activités quotidiennes avant la parution de l'épisode suivant.

10 L'analyse d'extraits des Mystères de Paris met cependant à mal un mythe persistant sur le feuilleton : le cliffhanger, qui nous semble aujourd'hui parfaitement évident et "naturel », et dont les feuilletonistes de la fin du siècle useront et abuseront quelques décennies plus tard, à l'instar de Ponson du Terrail, est loin d'être systématique à la fin des livraisons en 1842.

\section{Mise en place progressive des techniques narratives du feuilleton dans Les Mystères de Paris}

11 L'histoire éditoriale des Mystères nous permet de comprendre qu'ils se situent au début de leur publication dans une période de transition, encore marquée par la toute-puissance des éditeurs, avant l'avènement véritable du roman-feuilleton, qui triomphe précisément en partie grâce au succès d'Eugène Sue. Le contrat pour Les Mystères de Paris a été établi par l'éditeur d'Eugène Sue, Charles Gosselin: c'est lui qui négocie les conditions de publication dans Le Journal des Débats. En effet, malgré ses réticences initiales vis-à-vis du feuilleton, il comprend rapidement le surplus de bénéfices qui peut être tiré d'une double publication ${ }^{2}$. Les quatre premières parties, qui paraissent dans le journal entre juin et décembre 1842, ont donc été pré-écrites par Sue. À l'origine, la parution dans le journal est ainsi conçue comme une prépublication : la destination du roman est avant tout son édition en volumes, au format in-octavo, chez Gosselin, ce qui explique la présence d'un chapitrage en plus du découpage en feuilletons. Au terme de l'année 1842, Gosselin a pu mesurer le succès du roman et de nouvelles parties sont commandées, dont la composition épouse de plus près la parution dans le journal. La marge de temps de rédaction diminue : tandis que les cinquième et sixième parties sont écrites un mois avant leur publication, les dernières sont terminées seulement quelques jours avant, ce qui permet notamment à Sue de transformer son récit en véritable pamphlet réformiste et de participer pleinement au débat politique en réagissant avec virulence à l'actualité législative. Nous ne reviendrons pas ici sur ce phénomène de politisation, qui a été étudié par ailleurs (Thiesse 1980, Prendergast 2003, Grossir 2008, Lyon-Caen 2009, Thérenty 2014, Goudmand 2014), mais nous nous intéresserons à l'évolution du rythme narratif au fil du récit entre 1842 et 1843. 


\section{Feuilleton du 2 juillet 1842}

Dans le premier exemple que nous nous proposons d'étudier, le Prince Rodolphe a été enfermé dans une cave et prend conscience du terrible danger qui le menace : le niveau de l'eau est en train de monter :

Il écouta... il n'entendit rien... rien qu'une espèce de petit clapotement sourd, faible, mais continu.

D'abord il n'en soupçonna point la cause.

[...] Et au milieu du morne silence qui l'environnait, il entendit plus distinctement encore le petit clapotement sourd, faible, continu.

Cette fois, il en comprit la cause : l'eau envahissait le caveau...

[...] Rodolphe parcourut la cave à tâtons, en tous sens, ayant de l'eau jusqu'à mijambe ; il ne trouva rien. Il remonta l'escalier, dans un sombre désespoir.

[...] Il n'entendit rien, rien que le petit clapotement sourd, faible, continu, de l'eau qui toujours montait, montait, montait. (Sue, « Le caveau », 7, I, Journal des débats, 7 juillet $1842: 1)$

Le chapitre suit de près l'état psychologique de Rodolphe, qui alterne entre tentatives désespérées et inutiles de sortir du caveau, et moments d'accablement où il est envahi à la fois par le remords de ne pas avoir protégé son serviteur et par des rats qui cherchent à échapper à la noyade en se réfugiant sur ses vêtements. Après un dernier rebondissement dramatique, au moment où tout semble perdu, il est finalement sauvé par le Chourineur à la fin du chapitre :

Le vertige emportait la pensée de Rodolphe dans son rapide et effrayant tourbillon; l'eau bouillonnait à ses oreilles; il croyait se sentir tournoyer sur lui-même; la dernière lueur de sa raison allait s'éteindre, lorsque des pas précipités et un bruit de voix retentirent auprès de la porte de la cave.

L'espérance ramena ses forces expirantes ; par une suprême tension d'esprit, il put saisir ses mots, les derniers qu'il entendit et compris :

- Tu le vois bien, il n'y a personne.

- Tonnerre ! C'est vrai... répondit tristement la voix du Chourineur.

Et les pas s'éloignèrent.

Rodolphe, anéanti, n'eut pas la force de se soutenir davantage, il glissa le long de l'escalier.

Tout à coup, la porte du caveau s'ouvrit brusquement en-dehors; l'eau contenue dans le souterrain s'échappa comme par l'ouverture d'une écluse... et le Chourineur put saisir les deux bras de Rodolphe qui, à demi-noyé, se cramponnait encore au seuil de la porte par un mouvement convulsif. (Sue, "Le caveau », 7, I, Journal des débats, 7 juillet $1842: 1$ )

En tant que lecteur du Xxi ${ }^{e}$ siècle, on peut être surpris par le choix d'une telle coupe : on se serait plutôt attendu à ce que le chapitre se termine un paragraphe plus tôt pour un cliffhanger en bonne et due forme, laissant Rodolphe dans une position incertaine. Or, on a 
ici un chapitre qui s'achève sur un dénouement et qui, de plus, ne correspond pas à la fin de la livraison, puisque le chapitre suivant, intitulé "Le garde-malade ", paraît le même jour (illustration 2). On le voit donc, la technique du cliffhanger n'a rien d'intuitif, et elle n'est pas encore figée au moment où Sue écrit son livre (illustration 3). C'est qu'en 1842, Les Mystères de Paris sont avant tout conçus en fonction de l'édition en volumes. Voyons ce qu'il en est dans les épisodes ultérieurs.

Nous l'avons souligné plus haut, à partir de 1843 , le délai entre la rédaction et la publication dans le journal se raccourcit. Cette année marque ainsi un renversement de la logique initiale de Gosselin : le principe de l'écriture journalistique au jour le jour prend peu à peu le pas sur la disposition livresque. L'analyse de quelques exemples montrera les conséquences de ce changement de rythme sur la narration.

\section{Feuilletons des 12 et 13 mai 1843}

16 Fleur-de-Marie, pure héroïne que le prince Rodolphe a tirée de la prostitution, et figure de victime privilégiée, est régulièrement la cible des plans machiavéliques des méchants. Dans l'épisode publié dans Le Journal des Débats datant du 12 mai 1843, ses ennemis lui réservent un triste sort :

- Va-t-elle être contente cette chère dame! dit Mme Séraphin. Puis s'adressant à Nicolas : voyez mon garçon, approchez encore un peu plus votre bateau que nous puissions monter. Et elle ajouta tout bas : il faut absolument noyer la petite; si elle revient sur l'eau, replongez-la. (Sue, «Le bateau », 3, VII, Journal des débats, 12 mai $1843: 2)$

17 Les dernières lignes de la livraison, qui décrivent le départ des bateaux, ne résolvent pas la situation dysphorique mise en place, et le lecteur devra attendre les numéros suivants pour connaître le sort de la malheureuse. Si l'héroïne n'est pas encore en danger de mort au moment où le récit s'interrompt, l'intention des méchants n'en est pas moins claire : la situation d'urgence à laquelle fait face Fleur-de-Marie laisse le lecteur dans l'inquiétude et l'encourage à anticiper des scénarios possibles en attendant la résolution promise. Or, dans l'épisode du lendemain, le narrateur annonce une analepse qui va retarder le dénouement attendu et ouvrir une nouvelle sous-séquence centrée sur un autre personnage :

Avant d'apprendre au lecteur le dénouement du drame qui se passait dans le bateau à soupape de Martial, nous reviendrons sur nos pas. Peu de moments après que Fleur-de-Marie eut quitté Saint-Lazare avec Mme Séraphin, la Louve était aussi sortie de prison. (Sue, «Bonheur de se revoir », 4, VII, Journal des débats, 13 mai 1843, p.1)

18 Une fois sortie de prison, la Louve se rend sur l'île où Fleur-de-Marie a embarqué pour retrouver son amant Martial. Mais elle apprend que celui-ci a été emmuré vivant dans sa chambre par sa mère et son frère. La tension est alors à son comble, puisque la Louve est mobilisée sur deux fronts : elle doit sauver à la fois Fleur-de-Marie qui est en train de se noyer et son amant emprisonné. Le récit épouse le point de vue de la Louve qui apprend d'un vieux pêcheur ce qui est arrivé à son amant :

- [...] Me trouvant donc avec sa mère entre quatre-z-yeux, je n'ai pas osé éviter de lui parler [...]. « Voilà deux jours que je n'ai vu votre Martial, que je lui dis; il est donc parti en ville? ? Là-dessus elle me regarde avec des yeux... mais des yeux... qui m'auraient tué s'ils avaient été des pistolets, comme dit cet autre.

- Vous me faites bouillir. Après? Après? 
Le père Férot garda un moment le silence, puis reprit :

- Tenez, vous êtes une bonne fille, promettez-moi le secret, et je vous dirai toute la chose, comme je la sais.

- [...] Mais que se passe-t-il ? Qu'est-ce que sa mère et son frère lui ont fait ? Où estil ? Parlez donc, mais parlez donc !

- Allons, bon, vous voilà encore après ma blouse. Lâchez-moi donc! Si vous m'interrompez toujours en me détruisant mes effets, je ne pourrai jamais finir et vous ne saurez rien.

- Oh! Quelle patience! s'écria la Louve en frappant des pieds avec colère. (Sue, «Bonheur de se revoir », 4, VII, Journal des débats, 13 mai 1843, p. 3)

Ce passage est particulièrement intéressant dans le sens où il est révélateur de la dynamique interactionnelle du récit: les réactions impatientées de la Louve face à la réticence des révélations de son interlocuteur, qui retarde le dénouement pour ménager ses «effets", rejouent celles des lecteurs d'Eugène Sue qui attendent leur livraison quotidienne. La différence étant bien entendu que cette attente qui est un supplice pour la Louve se mue en plaisir pour le lecteur puisqu'elle est projetée sur un univers fictionnel: Baroni évoque à cet égard la différence entre la dysphorie passionnelle des expériences vécues et la dysphorie passionnante des histoires : «Dans l'espace du récit, les leçons que nous tirons habituellement des épreuves que nous réserve l'existence peuvent être enseignées sans danger: le vécu passionnel se transforme en histoire passionnante " (Baroni 2007 : 35). En outre, le lecteur sait que le temps est compté et que chaque moment perdu par la Louve est susceptible de mettre à mal le destin des personnages en danger. En l'occurrence, le lecteur ne partage pas complètement la lacune épistémique de la protagoniste : il sait que Martial a été attaqué par sa famille, mais il ne sait pas ce qu'il est advenu de lui. En effet, le feuilleton du 19 mars 1843 s'interrompait en laissant Martial et ses jeunes frère et sœur dans une posture tout aussi fâcheuse que celle de Fleur-deMarie :

François se rendit, quoique à regret, au désir de sa sœur, entrebâilla la persienne et regarda.

- Eh bien! mon frère ? dit Amandine en surmontant ses craintes et en s'approchant de François sur la pointe du pied. [...]

- Nicolas monte à l'échelle, il a sa hâchette à la main, je la vois reluire...

- Ah ! vous n'êtes pas couchés et vous nous espionnez ! s'écria tout à coup la veuve, en s'adressant du dehors à François et Amandine.

$\mathrm{Au}$ moment de rentrer dans la cuisine, elle venait d'apercevoir la lueur qui s'échappait de la persienne entrouverte.

Les malheureux enfants avaient négligé d'éteindre leur lumière.

- Je monte, ajouta la veuve d'une voix terrible, je monte vous trouver, petits mouchards! (Sue, «François et Amandine », 4, VI, Journal des débats, 19 mars 1843, p.3) 
ecteur sait que Fleur-de-Marie est également en danger, alors que la Louve n'en a aucune idée. L'économie du suspense est ici favorisée par le choix du point de vue, qui amène le lecteur à épouser par identification l'anxiété et l'impatience de la Louve, avide de connaître le fin mot de l'histoire : il y a concurrence entre l'urgence de la situation de Martial et la volonté du vieux pêcheur de prendre le temps de bien raconter ce qui s'est passé. Le pêcheur est donc un bon conteur, puisqu'il a bien compris que l'art du récit consiste précisément à retarder le dénouement attendu, mais un piètre adjuvant, puisqu'il fait perdre un temps précieux à la Louve. Dans la suite de l'épisode, au terme d'une véritable course contre la montre, et grâce à des capacités physiques quasiment surhumaines (et d'ailleurs rarement déployées par un personnage féminin dans la littérature de l'époque), la Louve sauve successivement Fleur-de-Marie, qui se noyait opportunément sur son chemin, et Martial, ce qui constitue le dénouement d'une première sous-séquence concernant ce dernier, dont le sort était resté en suspens depuis la livraison du 19 mars 1843, et de la sous-séquence concernant le sort de Fleur-de-Marie (illustration 4). Le feuilleton du 13 mai s'achève donc, contrairement au précédent, sur un dénouement provisoire :

Se jetant aussitôt à genoux dans le corridor, à l'aide du bec du merlin et de ses ongles qu'elle meurtrit, de ses doigts qu'elle déchira, la Louve parvint à arracher du plancher et du chambranle plusieurs clous énormes qui condamnaient la porte.

Enfin cette porte s'ouvrit.

Martial, pâle, les mains ensanglantées, tomba presque sans mouvement dans les bras de la Louve. (Sue, Journal des débats, 13 mai 1843, p.3)

21 Ici donc, et contrairement à l'exemple étudié précédemment, Sue alterne entre une clôture d'épisode suspensive, qui repousse le dénouement de la séquence et maintient l'intérêt du lecteur jusqu'à la livraison suivante, et une clôture conclusive, qui apporte la résolution des incertitudes. Le jeu des analepses et des prolepses permet de raconter des évènements qui ont lieu simultanément dans le temps de l'histoire, de multiplier les lieux d'incertitude et ainsi d'accroître la frustration et donc l'intérêt des lecteurs, qui ont été tenus en haleine sur des centaines d'épisodes. Mais cette attente n'est pas purement passive : elle est inséparable d'une activité anticipatrice qui mène les lecteurs à envisager les divers développements possibles pour la suite de l'histoire. Les lecteurs sont en outre confrontés à plusieurs niveaux d'incertitude quant au devenir des personnages: si on reprend l'exemple de Fleur-de-Marie, un premier niveau correspond à celui du temps court, de l'urgence, où elle doit se défendre, souvent sans succès, de ses nombreux agresseurs, tandis que le second niveau concerne son devenir existentiel sur le long terme, où les questions qu'elle se pose déterminent un scénario de vie plutôt qu'un autre (la vie de religieuse pour expier son passé de prostituée plutôt que celle de princesse, puis la mort plutôt que la vie de religieuse). De nombreux lecteurs ont fait partager à l'auteur leurs pronostics quant au destin de Fleur-de-Marie, en espérant parfois l'infléchir :

Maintenant, qu'allez-vous faire de Fleur-de-Marie, ne la faites pas mourir, nous l'aimons trop. Je ne sais quel sinistre pressentiment m'a saisie depuis son entrevue avec l'Ogresse, voudriez-vous priver Rodolphe de ce bonheur qu'il a si chèrement acheté, non, nous les reverrons sans doute tous les deux heureux à Gerolstein que nous attendons impatiemment. (Ernestine Dumont, in Lyon-Caen 2009 : 1274)

En plus de la fidélisation à court terme provoquée par " le magique "la suite à demain" ", pour reprendre l'expression de Gauthier, s'instaure ainsi une fidélisation sur du long terme qui dépend de l'attachement du public envers les personnages et l'univers 
diégétique, ce qui permet de maintenir l'intérêt même lorsque l'épisode se termine sur un dénouement provisoire.

\section{Feuilletons des 21,22 et 23 juin 1843}

L'exemple que nous étudierons pour finir se déroule en prison. Injustement enfermé, le malheureux Germain se retrouve entouré de criminels, dont le terrible Squelette et ses acolytes, qui projettent de l'assassiner depuis plusieurs chapitres. La fin du chapitre intitulé « Complot », publié le 16 juin 1843, qui se termine sur cette menace :

- Il va venir, prépare-toi.

- Je suis tout prêt ; il portera mes marques.

[...] - À la pâtée, les chiens! dit le Squelette; Pique-Vinaigre et Germain vont rentrer au préau. Attention, les amis, on m'appelle Mort-d'avance, mais le mangeur aussi est mort d'avance. (Sue, « Complot», 25, VII, Journal des débats, 16 juin 1843, p.3.)

Les comploteurs cherchent à détourner l'attention du gardien pour pouvoir mener à bien leur projet et comptent profiter pour cela du récit dans lequel se lance le dénommé Pique-Vinaigre pour divertir ses co-détenus :

Germain, Pique-Vinaigre et le gardien ignoraient seuls ce qui allait se passer.

L'attention générale se partageait entre le bourreau, la victime et le conteur qui allait innocemment priver Germain du seul secours que ce dernier pût attendre ; car il était presque certain que le gardien, voyant les détenus attentifs aux récits de Pique-Vinaigre, croirait sa surveillance inutile et profiterait de ce moment de calme pour aller prendre son repas. (Sue, «Le conteur », 26, VII, Journal des débats, 17 juin 1843, p.3)

C'est l'occasion pour l'auteur d'une réflexion sur le rôle éthique du récit de fiction, et plus précisément de l'empathie vis-à-vis des personnages fictifs :

Avant d'entamer le récit de Pique-Vinaigre, nous rappellerons au lecteur que, par un contraste bizarre, la majorité des détenus, malgré leur cynique perversité, affectionnent presque toujours les récits naïfs, nous ne voudrions pas dire puérils, où l'on voit, selon les lois d'une inexorable fatalité, l'opprimé vengé de son tyran, après des épreuves et des traverses sans nombre.

[...] On raille ordinairement ces incultes témoignages de sympathie pour ce qui est bon, faible et persécuté... d'aversion pour ce qui est puissant, injuste et cruel.

On a tort, ce nous semble.

Rien de plus consolant en soit que ces ressentiments de la foule.

N'est-il pas évident que ces instincts salutaires pourraient devenir des principes arrêtés chez les infortunés que l'ignorance et la pauvreté exposent incessamment à la subversive obsession du mal?

[...] L'impression causée par le récit de Pique-Vinaigre démontrera, ou plutôt exposera, nous l'espérons, quelques-unes des idées que nous venons d'émettre. (Sue, « Gringalet et Coupe-en-Deux », 27, VII, Journal des débats, 21 juin 1843. p.1)

En effet, le conte de Pique-Vinaigre, intitulé « Gringalet et Coupe-en-Deux », et conçu par Sue comme un petit apologue édifiant, a exactement l'effet inverse de celui qu'attendait 
le Squelette, puisqu'il captive aussi bien les prisonniers que le gardien, qui repousse sa pause déjeuner pour pouvoir écouter l'histoire :

À ce passage du récit de Pique-Vinaigre, l'horloge de la prison sonna trois heures un quart.

Les détenus rentrant dans les dortoirs à quatre heures, le crime du Squelette devait être consommé avant ce moment.

- Mille tonnerres ! le gardien ne s'en va pas, dit-il tout bas au Gros-Boiteux.

- Sois tranquille, une fois l'histoire en train, il filera...

Pique-Vinaigre continua son récit. (Sue, «Gringalet et Coupe-en-Deux », 27, VII, Journal des débats, 21 juin 1843. p.1)

À ce moment trois heures et demie sonnèrent.

Le bourreau de Germain et le Gros-Boiteux échangèrent un coup d'œil significatif. L'heure avançait, le surveillant ne s'en allait pas, et quelques-uns des détenus, les moins endurcis semblaient presque oublier les sinistres projets du Squelette contre Germain, pour écouter avec avidité le récit de Pique-Vinaigre. (Sue, « Gringalet et Coupe-en-Deux », 27, VII, Journal des débats, 21 juin 1843. p.2)

27 Ainsi, dans cette livraison et celle du lendemain, chaque rebondissement de l'histoire racontée par Pique-Vinaigre retient le gardien et retarde l'exécution du plan du Squelette. Le récit enchâssé raconte également une histoire de sauvetage: Gringalet, garçon sans défense qui ne supporte pas l'injustice dont sont victimes les plus faibles, passe son temps à secourir des moucherons sur le point d'être dévorés par des araignées. Alors qu'il est sur le point d'être tué par le brigand Coupe-en-Deux, par un juste retour des choses, il est sauvé par un moucheron qui se faufile dans l'œil de son bourreau. Les complices du Squelette identifient inconsciemment Gringalet à Germain, et leur volonté de nuire à un innocent faiblit. Mais la conclusion du récit est interrompue par le départ du gardien, laissant le champ libre au Squelette :

Quant à Gringalet, [...] il répétait tout le temps de son triomphe :

«- Petits moucherons, j'ai bien fait d'empêcher les araignées de vous manger, car...»

La fin du récit de Pique-Vinaigre fut interrompue.

- Eh! père Roussel, cria une voix de dehors, viens donc manger ta soupe ; quatre heures vont sonner dans dix minutes.

- Ma foi, l'histoire est à peu près finie, j'y vais. Merci, mon garçon, tu m'as joliment amusé, tu peux t'en vanter, dit le surveillant à Pique-Vinaigre en allant vers la porte. Puis, s'arrêtant : «Ah çà ! soyez sages », dit-il aux détenus en se retournant. (Sue, « Gringalet et Coupe-en-Deux », 27, VII, Journal des débats, 21 juin 1843. p.4)

La fin du feuilleton du 22 juin laisse Germain, victime d'une attaque sauvage du Squelette, en danger de mort, alors que les dialogues consacrent l'identification des personnages du récit cadre à ceux du récit enchâssé :

- À moi! Gringalet... Je serai ton araignée, s'écria aussitôt le Squelette en se précipitant si brusquement sur Germain, que celui-ci ne put faire un mouvement ni pousser un cri. 
Sa voix expira sous la formidable étreinte des longs doigts de fer du Squelette. (Sue,

"Le triomphe de Gringalet et Gargousse », Journal des débats, 22 juin 1843, p.4)

L'action est alors interrompue au moment où le suspense est le plus insoutenable : Eugène Sue s'est adapté à la parution journalière et laisse son lecteur sur un cliffhanger précoce. Cette succession de chapitres est donc structurée par un suspense à deux niveaux : celui du récit cadre et celui du récit mis en abyme, qui finissent par s'entremêler. Germain est tiré d'affaire au début du chapitre suivant, toujours grâce aux bons soins de l'héroïque Chourineur, qui permet un dénouement conforme à l'histoire de Gringalet et Coupe-enDeux :

XXVIII. Un ami inconnu

- Si tu es l'araignée, moi je serai le moucheron d'or, Squelette de malheur, cria une voix au moment où Germain, surpris par la violente et soudaine attaque de son implacable ennemi, tombait renversé sur son banc, livré à la merci du brigand qui, un genou sur la poitrine, le tenait par le cou. [...] Puis, d'un bond furieux, renversant trois ou quatre prisonniers qui le séparaient de Germain, il s'élança sur le Squelette et lui asséna sur le crâne et entre les deux yeux une grêle de coups de poing si précipitée, qu'on eût dit la batterie sonore d'un marteau sur une enclume. (Sue, « Un ami inconnu », 29, VII, Journal des débats, 23 juin 1843, p.1)

L'histoire de Gringalet a un impact immédiat sur le « réel » (de la fiction) : d'une part elle retarde l'attaque de Germain par le Squelette ; d'autre part elle agit sur le comportement des détenus qui apprennent à s'identifier à autrui et qui prennent fait et cause pour Germain: chaque nouveau rebondissement dans le récit enchâssée est la cause d'un retardement de l'action dans le récit cadre. Le récit enchâssé est dénoué avant la fin de l'épisode, contrairement à la séquence du récit cadre (voir illustration 5). Ainsi, Sue cherche à la fois à revaloriser sa position de feuilletoniste en montrant, exemplum gratia, les vertus morales de la fiction et offre du même coup une double ration de suspense à son lecteur en lui proposant, en quelque sorte, deux récits pour le prix d'un. Mesurons l'effet de ce passage sur un lecteur enthousiaste qui écrit à Eugène Sue :

Vous êtes donc sorcier, Monsieur, pour me faire éprouver tout ce que j'ai éprouvé depuis quelques heures, pour m'avoir mis dans l'état où je suis en ce moment; j'ai eu la chair de poule pour le pauvre Germain pendant le récit de Pique-Vinaigre, et puis ma poitrine s'est dilatée, en accompagnant (par la pensée) la dégelée de coups de poings de la fin que notre bon Chourineur festonnait si bien sur la sorbonne du Squelette [...]. (Adrien Decourcelle, in Galvan 1998, II : 298, lettre 378)

Selon David Lodge (1992), le cliffhanger ne sera développé à proprement parler qu'une trentaine d'années après la parution des Mystères, par Thomas Hardy puis Wilkie Collins. Si cette date se vérifie peut-être dans le contexte anglophone, un regard sur le domaine francophone encourage à l'anticiper (quand bien même le terme effectif n'existe pas encore) et permet comprendre les différentes fonctions qu'il remplit dans le romanfeuilleton. L'artifice des effets narratifs du roman-feuilleton a souvent été dénoncé par ses détracteurs, pourtant cette alternance entre continuité et discontinuité, entre temps court et temps long de l'intrigue ne saurait se réduire à un simple procédé commercial : elle correspond à l'expérience de la temporalité telle que nous l'éprouvons au quotidien. L'anticipation des scénarios possibles, qui articule cognition et émotion, a une valeur mimétique et modélisatrice (Schaeffer 1999); elle permet de convertir en plaisir des expériences dysphoriques d'incertitude, et ainsi de mieux les appréhender dans la vie réelle. Dans le cas des Mystères de Paris, et de bien d'autres romans-feuilletons, nous nous trouvons en présence d'une littérature qui, plutôt que la postérité, vise à être en prise 
avec le plaisir immédiat des lecteurs contemporains, ce qui nous amène à prendre en compte l'ordinaire de l'expérience de lecture si souvent disqualifié.

\section{BIBLIOGRAPHY}

Baroni, R., (2004), « La valeur littéraire du suspense », A contrario, vol. 2, p. 29-43.

Baroni, R. (2007), La Tension narrative. Suspense, curiosité et surprise, Paris : Seuil, « Poétique ».

Dionne, U. (2008), La voie aux chapitres. Poétique de la disposition romanesque, Paris : Seuil,

«Poétique ».

Dumasy, L. (1999), La querelle du roman-feuilleton: Littérature, presse et politique, un débat précurseur (1836-1848), Grenoble : Ellug.

Galvan ; J.-P. (éd.) (1998), Les Mystères de Paris : Eugène Sue et ses lecteurs, 2 tomes, Paris :

Harmattan.

Gautier, T. (1844), feuilleton dramatique de La Presse, 19 février 1844.

Goudmand, A. (2014) «L'ethos du feuilletoniste : interventions d'auteur chez Eugène Sue », in Meizoz, J., Mühlethaler, J.-C., \& Burghgraeve, D. (dir.), Actes du colloque « Figures, ethos et postures d'auteurs au fil des siècles ", Fabula, la recherche en littérature, colloques en ligne, URL : http://www.fabula.org/colloques/document2344.php.

Grossir, Cl. (2008), « Du feuilleton à l'Assemblée Nationale : Eugène Sue et Les Mystères de Paris ", Romantisme, ${ }^{\circ} 141$, p.107-118.

Guise, R. (1964), « Balzac et le roman-feuilleton », L’Année balzacienne, p. 283-338.

Guise, R. (1992), «Les Mystères de Paris, histoire d'un texte. Légende et vérité », Bulletin des amis du roman populaire, $\mathrm{n}^{\circ} 17$, p. 9-29.

Iser, W. (1976), L’Acte de lecture. Théorie de l'effet esthétique, Bruxelles : Pierre Mardaga.

Lodge, D. (1992), The Art of Fiction, London : Penguin.

Messac, R. (2011 [1929]), Le « Detective Novel » et l'influence de la pensée scientifique, nouvelle édition revue, Paris : les Belles lettres, « Encrage ».

Prendergast, Ch. (2003), For the People by the People? Eugene's Sue Les Mystères de Paris. A Hypothesis in the Sociology of Sitterature, Oxford : Legenda.

Reybaud, L. (1844 [1842]), Jérôme Paturot à la recherche d'une position sociale, Paris : Paulin.

Schaeffer, J.-M. (1999), Pourquoi la fiction?, Paris : Seuil, « Poétique ».

Sue, E. (2009 [1842-1843]), Les Mystères de Paris, Lyon-Caen J. (éd.), Paris : Gallimard.

Thérenty, M.-È. et Vaillant, A. (2001), 1836 : L’An I de l'ère médiatique, étude littéraire et historique du journal La Presse, d'Émile de Girardin, Paris : Nouveau Monde éditions.

Thérenty, M.-È. (2014), « Les mystères urbains ou triomphes et échecs de la fiction politique au XIX ${ }^{\mathrm{e}}$ siècle », Raison-publique.fr, URL : http://www.raison-publique.fr/article696.html. 
Thiesse, A.-M. (1980), « L'Éducation sociale d'un romancier : le cas d'Eugène Süe », Actes de la recherche en sciences sociales, vol. 32-33, p. 51-63.

\section{APPENDIXES}

Illustration 1

\section{Le cliffhanger}

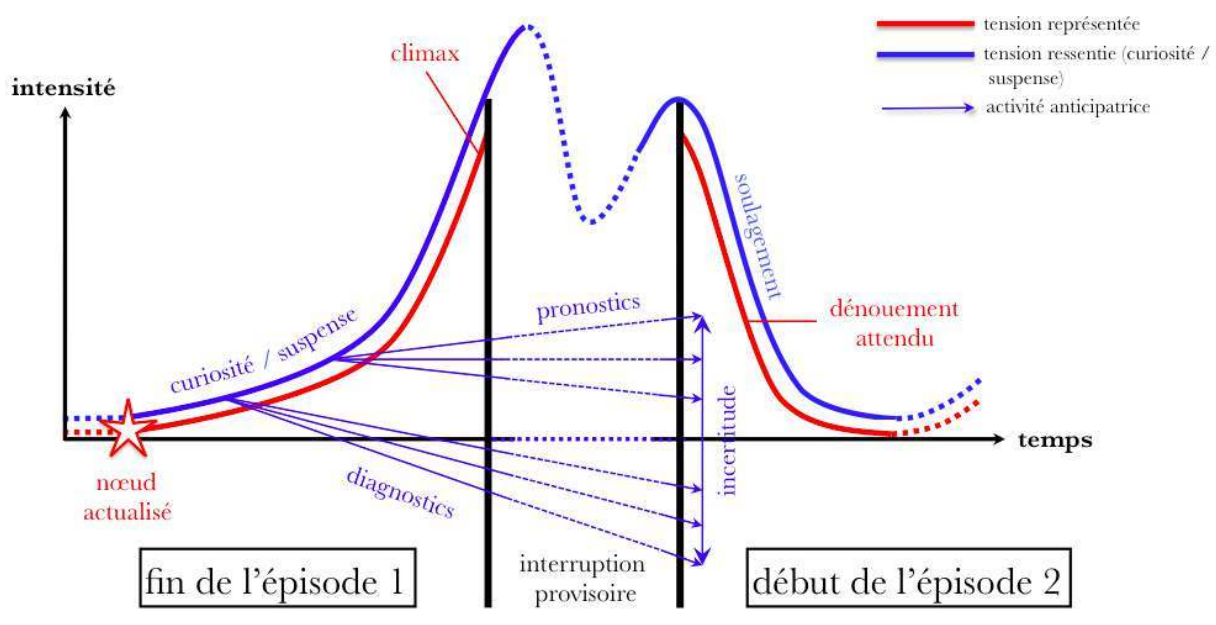

Schéma inspiré par « La courbe de la tension narrative » Baroni 2007: 131

\section{Illustration 2}

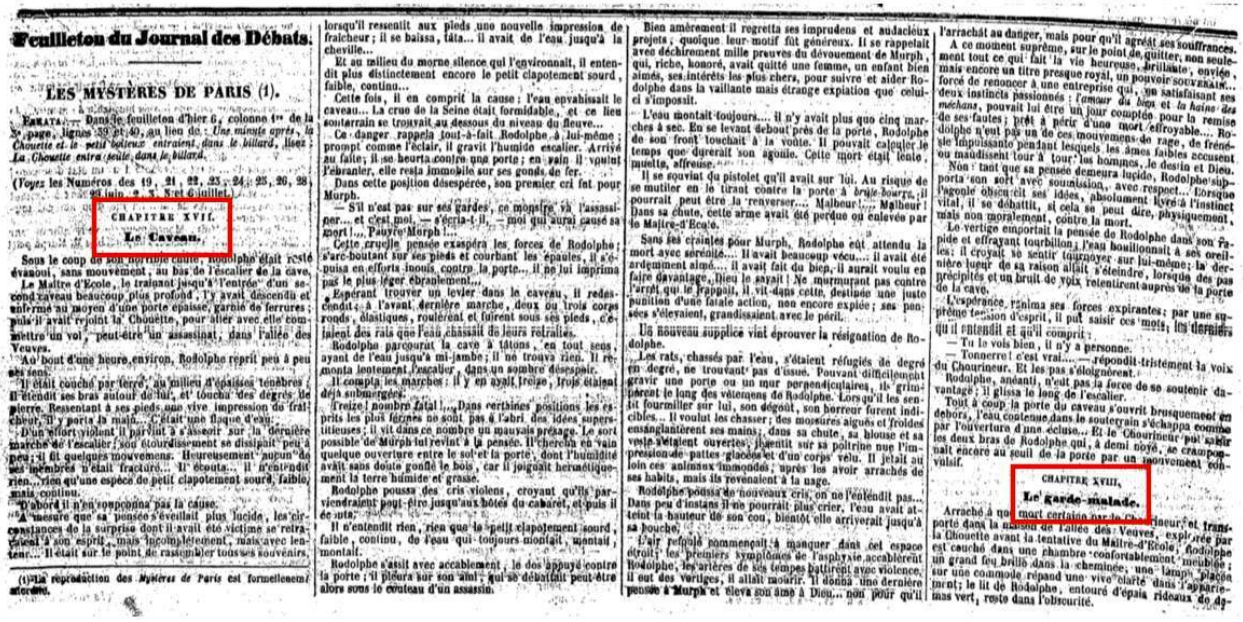

Journal des Débats, 7 juillet 1842, p.1. Source : www.gallica.bnf.fr 
Illustration 3

\section{Les Mystères de Paris, feuilleton du 2 juillet 1842}

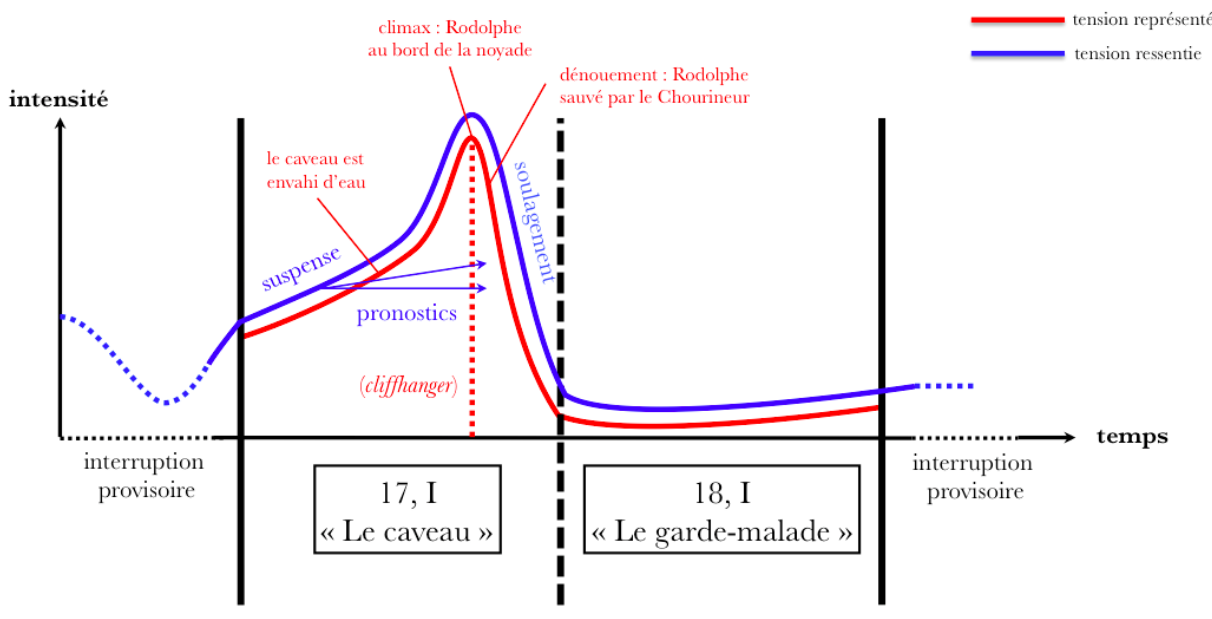

Illustration 4

Les Mystères de Paris, feuilletons des 12 et 13 mai 1843

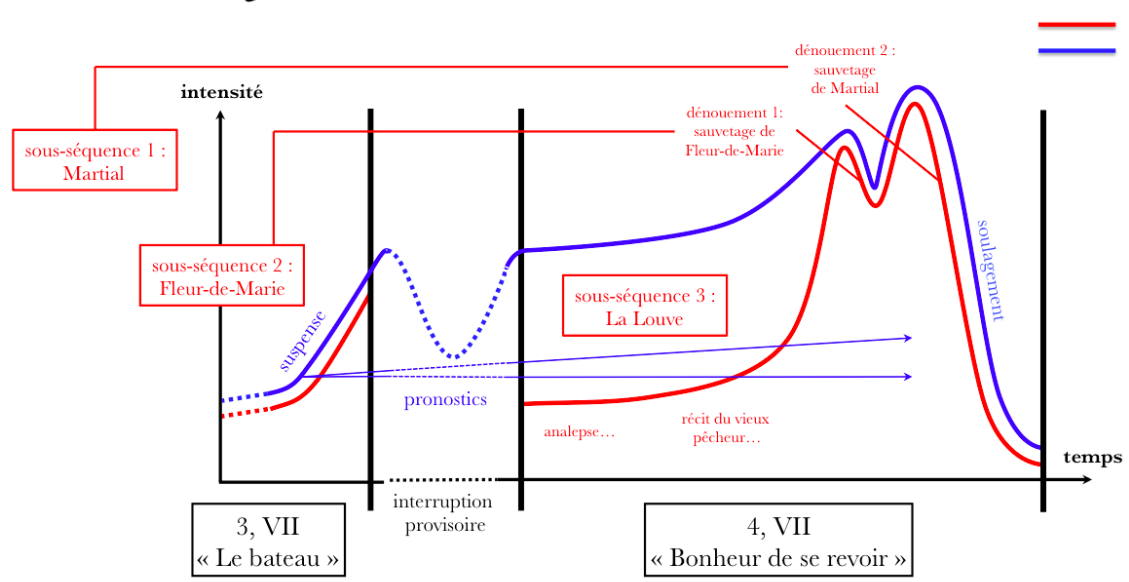




\section{Les Mystères de Paris, feuilletons des 22 et 23 juin 1843}

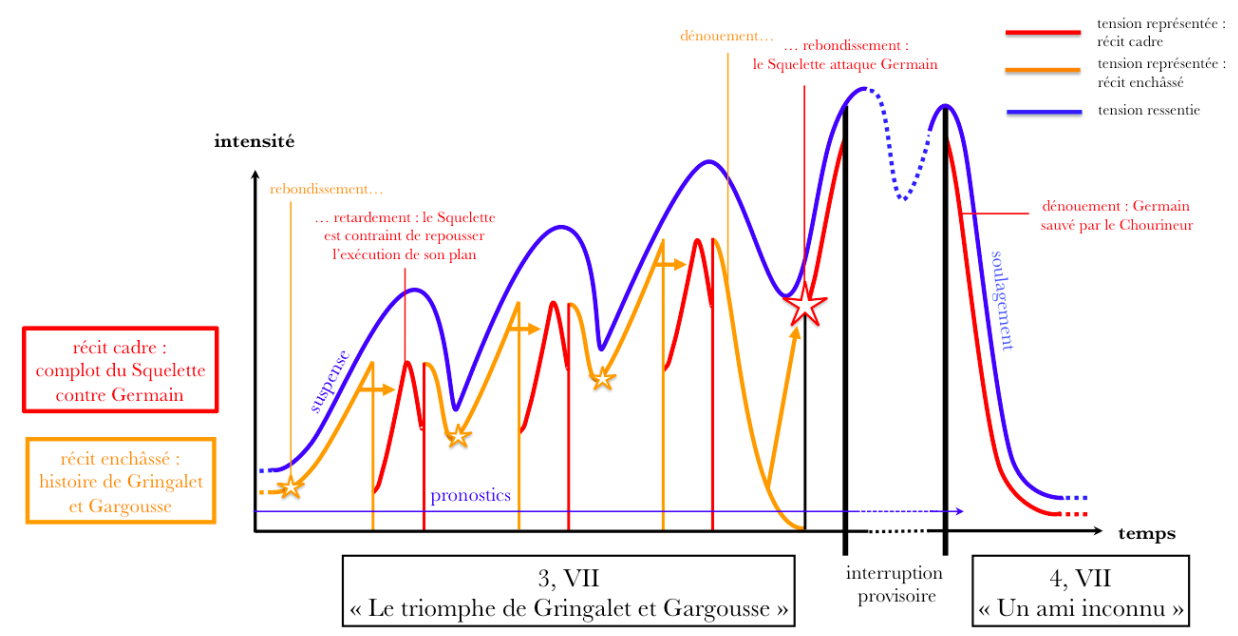

\section{NOTES}

1. Rappelons que dans les années 1840 , le journal ne s'achète pas au numéro : seuls les abonnés peuvent y avoir accès. Le but est d'inciter le lecteur non pas à acheter la livraison suivante, mais à s'abonner.

2. Pour une étude plus poussée du processus de rédaction des Mystères de Paris, voir Guise 1992 : 9-29, et Galvan 1998 : 20.

\section{ABSTRACTS}

This paper aims at analysing the narrative consequences of daily publication by studying the means of narrative tension in the serial organization, with particular emphasis on the example of Les Mystères de Paris by Eugène Sue, which were published between 1842 and 1843 in Le Journal des débats.

Cet article vise d'analyser les conséquences narratives de la publication en tranches quotidiennes à travers l'étude des modalités de la tension narrative en régime feuilletonnesque, en s'attachant plus particulièrement à l'exemple des Mystères de Paris d'Eugène Sue, parus entre 1842 et 1843 dans Le Journal des débats. 
INDEX

Geographical index: France

Chronological index: XIXe siècle

Mots-clés: roman-feuilleton, Eugène Sue, narratologie, cliffhanger

\section{AUTHOR}

\section{ANAÏS GOUDMAND}

Centre de Recherche sur les Arts et le Langage (CRAL) de l'EHESS - Université de Lausanne

Agrégée de Lettres Modernes, ancienne élève de l'École Normale Supérieure de Paris, Anaïs Goudmand est actuellement doctorante au Centre de Recherche sur les Arts et le Langage (CRAL) de l'EHESS sous la direction de Jean-Marie Schaeffer et à l'Université de Lausanne, en Suisse sous la direction de Raphaël Baroni (intitulé du sujet : « Sérialité et fictionalité : pour une poétique du récit sériel ») et assistante au département de Français Langue Étrangère à l'Université de Lausanne. 